\title{
Is early definitive fixation of bicondylar tibial plateau fractures safe? An observational cohort study
}

Unno, Florence ; Lefaivre, Kelly A ; Osterhoff, Georg ; Guy, Pierre ; Broekhuyse, Henry M ; Blachut, Piotr A ; O Brien, Peter

\begin{abstract}
OBJECTIVES: The optimal treatment protocol for bicondylar plateau fractures remains controversial. Contrary to popular practice which favors a staged protocol in many high-energy fracture patterns, we have used early single-stage open reduction and internal fixation (ORIF) to treat these injuries whenever possible. The purpose of this study was to determine the complication rate and the functional and radiographic outcomes of this strategy. DESIGN: Retrospective cohort study and prospective data collection. SETTING: Level I trauma center. PATIENTS/PARTICIPANTS: One hundred one patients with 102 OTA/AO type 41-C bicondylar tibial plateau fractures were treated with early definitive ORIF, defined as nonstaged surgery performed within 72 hours from injury. A subset of patients was part of a longitudinal study and reported functional outcomes at 1 year. INTERVENTION: Early definitive ORIF. MAIN OUTCOME MEASUREMENT: Primary outcome: reoperation rate, defined as any surgery within 12 months after the index operation; secondary outcomes: quality and stability of radiographic fracture reduction; and functional outcome [Medical Outcomes Study 36-Item Short-Form Health Survey (SF-36) and short musculoskeletal functional assessment (SMFA)]. RESULTS: Nonstaged operative treatment of bicondylar plateau fractures was performed in $91.3 \%$ of the fractures during the study period. For those, early definitive ORIF (surgery within 72 hours from injury) was performed in $82.3 \%$ fractures. Mean time from injury to ORIF, for closed fractures, was 29.8 hours. Sixteen (15.7\%) fractures, which were treated with early definitive ORIF, required an additional surgical procedure within 12 months. Complications included wound infection requiring surgical management, compartment syndrome requiring fasciotomies, nonunion, early fixation failure, and implant removal for discomfort. The reoperation rate was $12.7 \%$ if implant removal was excluded. At least 3 of the 4 radiographic criteria used to assess the adequacy of reduction were achieved in $95.1 \%$ of cases, and all 4 criteria were met in $59.8 \%$ of fractures. The Physical Component of the SF-36 at 12 months was 42.6, which is comparable to values reported in previous studies for operative treatment of bicondylar plateau fractures. CONCLUSIONS: In a model where surgery is performed without delay by experienced orthopaedic trauma surgeons, a large proportion of bicondylar tibial plateau fractures can be safely treated with early definitive ORIF. Early surgery was associated with satisfactory postoperative radiographic reductions. LEVEL OF EVIDENCE: Therapeutic Level IV. See Instructions for Authors for a complete description of levels of evidence.
\end{abstract}

DOI: https://doi.org/10.1097/BOT.0000000000000779

Posted at the Zurich Open Repository and Archive, University of Zurich

ZORA URL: https://doi.org/10.5167/uzh-140514

Journal Article

Published Version 
Originally published at:

Unno, Florence; Lefaivre, Kelly A; Osterhoff, Georg; Guy, Pierre; Broekhuyse, Henry M; Blachut, Piotr A; O Brien, Peter (2017). Is early definitive fixation of bicondylar tibial plateau fractures safe? An observational cohort study. Journal of Orthopaedic Trauma, 31(3):151-157.

DOI: https://doi.org/10.1097/BOT.0000000000000779 


\title{
Is Early Definitive Fixation of Bicondylar Tibial Plateau Fractures Safe? An Observational Cohort Study
}

\author{
Florence Unno, MD, MA, FMH, Kelly A. Lefaivre, MD, MSc, FRCSC, Georg Osterhoff, MD, FMH, \\ Pierre Guy, MD, MBA, FRCSC, Henry M. Broekhuyse, MD, FRCSC, Piotr A. Blachut, MD, FRCSC, \\ and Peter O'Brien, MD, FRCSC
}

\begin{abstract}
Objectives: The optimal treatment protocol for bicondylar plateau fractures remains controversial. Contrary to popular practice which favors a staged protocol in many high-energy fracture patterns, we have used early single-stage open reduction and internal fixation (ORIF) to treat these injuries whenever possible. The purpose of this study was to determine the complication rate and the functional and radiographic outcomes of this strategy.
\end{abstract}

Design: Retrospective cohort study and prospective data collection.

Setting: Level I trauma center.

Patients/Participants: One hundred one patients with 102 OTA/ AO type 41-C bicondylar tibial plateau fractures were treated with early definitive ORIF, defined as nonstaged surgery performed within 72 hours from injury. A subset of patients was part of a longitudinal study and reported functional outcomes at 1 year.

Intervention: Early definitive ORIF.

Main Outcome Measurement: Primary outcome: reoperation rate, defined as any surgery within 12 months after the index operation; secondary outcomes: quality and stability of radiographic fracture reduction; and functional outcome [Medical Outcomes Study 36-Item Short-Form Health Survey (SF-36) and short musculoskeletal functional assessment (SMFA)].

Results: Nonstaged operative treatment of bicondylar plateau fractures was performed in $91.3 \%$ of the fractures during the study period. For those, early definitive ORIF (surgery within 72 hours from injury) was performed in $82.3 \%$ fractures. Mean time from injury to ORIF, for closed fractures, was 29.8 hours. Sixteen $(15.7 \%)$ fractures, which were treated with early definitive ORIF, required an additional surgical procedure within 12 months.

Accepted for publication December 20, 2016.

From the Department of Orthopaedics, Division of Orthopaedic Trauma, University of British Columbia, Vancouver, BC, Canada.

K. A. Lefaivre, P. Guy, H. M. Broekhuyse, P. A. Blachut, and P. O'Brien receive institutional research support from Synthes and Zimmer. F. Unno and $\mathrm{K}$. A. Lefaivre received a research grant from AONA to support this work. The remaining authors report no conflict of interest.

Presented at the Annual Meeting of the Orthopaedic Trauma Association, October 9, 2015, San Diego, CA.

Reprints: Florence Unno, MD, MA, FMH (Foederatio Medicorum Helveticorum), Department of Orthopaedics, Division of Orthopaedic Trauma, University of British Columbia, VGH Research Pavilion 110-828 West 10th Avenue, Vancouver, BC V5Z 1M9, Canada (e-mail: fuvsen@gmail.com).

Copyright (C) 2016 Wolters Kluwer Health, Inc. All rights reserved.

DOI: 10.1097/BOT.0000000000000779
Complications included wound infection requiring surgical management, compartment syndrome requiring fasciotomies, nonunion, early fixation failure, and implant removal for discomfort. The reoperation rate was $12.7 \%$ if implant removal was excluded. At least 3 of the 4 radiographic criteria used to assess the adequacy of reduction were achieved in $95.1 \%$ of cases, and all 4 criteria were met in $59.8 \%$ of fractures. The Physical Component of the SF-36 at 12 months was 42.6, which is comparable to values reported in previous studies for operative treatment of bicondylar plateau fractures.

Conclusions: In a model where surgery is performed without delay by experienced orthopaedic trauma surgeons, a large proportion of bicondylar tibial plateau fractures can be safely treated with early definitive ORIF. Early surgery was associated with satisfactory postoperative radiographic reductions.

Key Words: tibia, plateau, fracture, complications, outcome

Level of Evidence: Therapeutic Level IV. See Instructions for Authors for a complete description of levels of evidence.

(J Orthop Trauma 2017;31:151-157)

\section{INTRODUCTION}

Operative management of bicondylar tibial plateau fractures remains challenging. Concomitant damages, fracture comminution, and soft-tissue injuries complicate the treatment of these high-energy injuries, which are consequently associated with adverse events and morbidity for the patients. ${ }^{1-3}$

The historical evolution of expert opinion on internal fixation for bicondylar tibial plateau fractures has mirrored that of pilon fractures. Despite favorable reports for early definitive care of pilon fractures presented by a leading group in Switzerland in the late 1970s, ${ }^{4}$ attempts at reproducing those results in North America proved dismal. Similarly, reports describing acute open reduction and internal fixation (ORIF) of high-energy proximal tibia fractures with dual plating in the 1980s and 1990s were associated with rates of infection as high as $87.5 \%{ }^{5-9}$ Those concerning results, combined with successful outcomes contemporaneously obtained with the use of temporizing external fixators for pilon injuries, ${ }^{10}$ contributed to the widespread adoption of staged protocols for the treatment of tibial plateau fractures. ${ }^{11,12}$

However, a critical review of early publications reporting high infection rates for acute fixation of high-energy tibial 
plateau fractures reveals that reports consist of small series, describing perioperative management and surgical techniques which would no longer be considered appropriate today-notable examples being the absence of antibiotic prophylaxis or the use of a midline incision. ${ }^{6,8,9}$ More recent case series with small patient numbers have suggested that modern operative techniques have decreased the risks associated with early definitive care. Benirschke et $\mathrm{al}^{13}$ reported no deep infection nor implant loosening after immediate ORIF of 14 open Gustilo grade II and IIIA complex tibial plateau fractures. Eggli et al ${ }^{14}$ presented good radiographic reduction and functional outcome with no infections in 14 bicondylar tibial plateau fracture treated with primary ORIF using dual incisions in all but 1 case. Eleven of 14 patients were operated within the first 24 hours after injury.

In our institution, we believe that operative treatment, performed as early as possible after injury, can be performed without increasing the number of postoperative complications. We have therefore continued to perform early definitive ORIF of lower extremity articular fractures whenever possible.

The goal of our study was to determine the safety of early fixation for bicondylar tibial plateau fractures. We hypothesized that definitive ORIF, performed early by subspecialty-trained orthopaedic trauma surgeons, results in an acceptably low rate of complications with satisfactory radiographic and functional outcomes.

\section{PATIENTS AND METHODS}

This report follows the STROBE guidelines for the reporting of observational studies. ${ }^{15}$ Using a prospectively collected orthopaedic trauma database, we identified 157 patients with 161 Arbeitsgemeinschaft für Osteosynthesefragen/ Orthopaedic Trauma Association type 41-C fractures, operatively treated in our level 1 trauma center between January 2004 and September 2014.

Fourteen fractures in 12 patients were staged and were therefore excluded ( 9 were treated with a temporizing external fixator in our institution; 5 were referred to our hospital after initial placement of an external fixator). One of those patients remained in the study group, as she sustained bilateral injuries with 1 side treated with early definitive ORIF. The choice of timing and surgical protocol (primary ORIF vs. staged protocol) was left at the discretion of the attending surgeon. As a general rule, the use of spanning fixators was limited to those cases where the presence of circumferential blisters, extensive contusion or necrosis, or the general condition of the patient made early fixation unreasonable. In particular, the absence of the "skin fold sign" was not retained as contraindication to early definitive surgery.

Patients who were not treated with a staged protocol with an external fixator were all treated with definitive ORIF: no other surgical modalities, such as rings or hybrid fixation, were recorded.

We further excluded 26 patients with 26 fractures operated after the 72-hour timeframe, which was arbitrarily chosen to define early definitive fixation. Nineteen patients with 19 fractures had insufficient follow-up and were excluded.

Our study population therefore consisted of 102 fractures in 101 patients, who were treated with early definitive ORIF, with a clinical follow-up of 12 months or more. Of the 101 patients, a subset of patients, starting in 2005, provided their informed consent to be enrolled in a prospective study to determine the functional outcome in these injuries (ethics' committee certificate H04-70551). Sufficient data were available for 31 of those patients.

All data were abstracted from the electronic medical records, admission and follow-up notes, operative notes, and radiographs. Patient demographics and potential risk factors including age, sex, comorbidities, and injury severity score (ISS) on admission were recorded. Open fractures were classified according to Gustilo and Anderson. ${ }^{16,17}$ Fractures were divided into 41-C1, 41-C2, or 41-C3 types using the OTA/AO classification. ${ }^{18}$ Treatment variables such as the timing of the initial surgery from injury and admission, the cause and nature of subsequent surgeries for complications, and the type and number of approaches were recorded.

\section{Outcome Measures}

The primary outcome was the reoperation rate within 12 months of the initial surgery. Any additional surgical interventions were recorded and included: fasciotomies for compartment syndrome after surgery, revision ORIF, operative debridement of a deep infection or wound dehiscence, treatment for nonunion, and removal of implant for discomfort.

Secondary outcome measures were radiographic reduction and stability and functional outcome. The quality of the reduction was determined based on analysis of biplanar radiographs obtained postoperatively. It was evaluated using 4 criteria described in previous studies ${ }^{19-25}$ : (1) an articular reduction with less than a 2-mm step or gap at the joint; (2) a medial proximal tibial angle (MPTA) of $87 \pm 5$ degrees for coronal alignment; (3) a posterior proximal tibial angle (PPTA) of $9 \pm 5$ degrees for sagittal alignment; and (4) condylar width difference of less than $5 \mathrm{~mm}$ compared with the femoral condyles. Two board-certified trauma-trained orthopaedic surgeons independently assessed the anteroposterior and lateral postoperative radiographs. These radiographs were analyzed using digital imaging software (iSite PACS Phillips) after correction for image magnification. Each measurement was taken 3 times and the average measurement was retained for the study. The same method was used when analyzing follow-up radiographs to evaluate the stability of reduction.

For the subset of patients who were prospectively followed, functional outcome was measured by a general health survey (the Medical Outcomes Study 36-Item ShortForm Health Survey) and a musculoskeletal questionnaire [the short musculoskeletal functional assessment (SMFA)]. The SF-36 is a widely accepted general health status measure of patients with a musculoskeletal condition. ${ }^{26}$ The SMFA, scaled from 0 to 100 with higher scores indicating poorer function, is a valid, reliable, and responsive outcome measure recommended by the American Academy of Orthopaedic Surgeons. $^{27-29}$ It comprises 2 indices: the Dysfunction Index (DI), which has 34 items for the assessment of patient function, and the Bother Index, which has 12 items for the assessment of how much patients are bothered by functional problems. Questionnaires were administered close to the time of admission, and baseline values were obtained by asking the 
participants to "recall" their preonset health status. Subsequent questionnaires were administered at 6 months and 1 year as a part of the prospective study protocol.

\section{Statistical Analysis}

Statistical analysis was performed using SPSS 22.0 (SPSS Inc, Chicago, IL). Nominal variables were associated using $\chi^{2}$ or Fisher exact tests. Continuous and categorical data were compared using Student $t$ test with a Levene test of variance in case of normal distributions. A nonparametric analysis was used in case of nonnormally distributed continuous and nominal data. Correlations between continuous data were assessed using Pearson correlation coefficient. The level of significance was defined as $P<0.05$.

\section{RESULTS}

In the 101 patients (102 fractures) with OTA 41-C fractures treated with early definitive ORIF, 53 were male and 48 were female. The mean age was 51 years (range, 1986 years). Mechanisms of injury included falls (40), pedestrians struck by a motor vehicle (26), motor vehicle accidents (10), motorcycle accidents (10), bicycle accidents (5), a direct blow (4), a twisting mechanism (3), a crush injury (2), and an all-terrain vehicle accident (1). There were 97 closed fractures and 5 open fractures (type I $n=2$; type II $n=2$, and type IIIA $n=1$ ). The mean ISS was 11.2 (range, 9-50), and 12 patients $(11.8 \%)$ had an ISS $>15$. There was no significant difference in age $(P=0.123)$ and sex $(P=0.253)$ between patients treated with early definitive ORIF and patients excluded from our study who were treated with a staged protocol. Patients with early definitive ORIF had a lower ISS score than patients with a staged protocol $(11$ vs. $17, P=$ $0.024)$, and patients with an open fracture were more likely to be treated with a staged protocol $(28.6 \%$ vs. $5.0 \%, P=0.012$; note, however, the small number of open fractures in both groups (total $\mathrm{n}=9$ ).

\section{Timing of Surgery}

Patients with closed injuries were operated within 24 hours after injury in $52.6 \%$ of cases and within 48 hours in $84.5 \%$ (Fig. 1). The mean time from injury to ORIF was 29.8 hours (median 23.2). The average time from injury to ORIF for patients with open fractures was 28.6 hours.

\section{Reoperation Within 12 Months for Complications}

Sixteen (15.7\%) fractures were reoperated within 12 months, with a total of 28 additional surgeries. Eleven patients underwent 1 surgery after their initial ORIF; 2 patients underwent 2 surgeries; 1 patient underwent 3 surgeries; and 2 patients underwent 5 surgeries (Table 1).

The overall rate of infection requiring surgical management was $8.8 \%(\mathrm{n}=9)$; this rate was $40.0 \%$ in open fractures ( 2 of 5 fractures) and $7.2 \%$ in closed fractures (7 of 97 fractures). Reoperation to perform a fasciotomy was required in 3 cases: 1 patient was diagnosed with compartment syndrome later during the same day after his surgery for ORIF; 1 patient developed a compartment syndrome 1 day

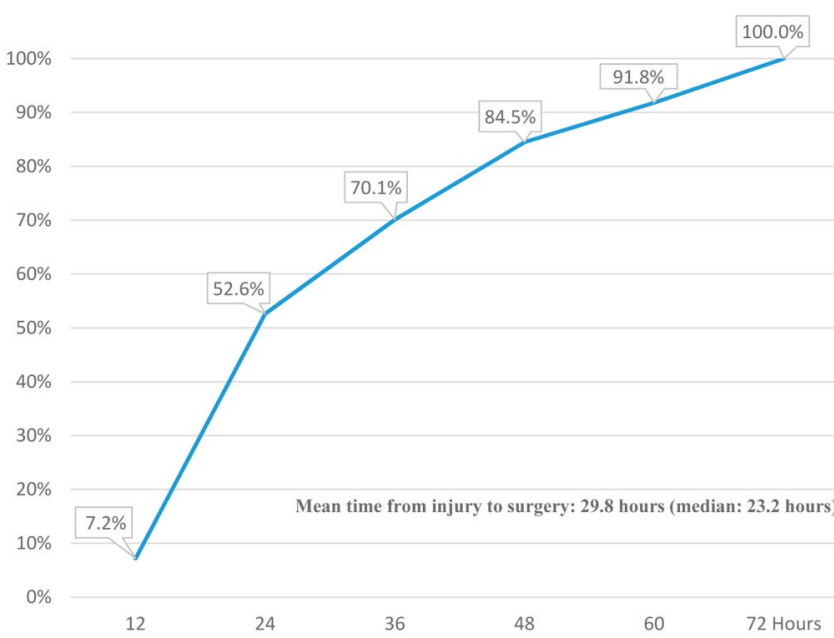

FIGURE 1. Proportion of patients operated after injury for closed fractures-cumulative incidence curve. Editor's Note: A color image accompanies the online version of this article.

postoperatively and another at 2 days. All 3 patients were emergently operated with fasciotomies followed by closure 5 days after the index surgery (primary closure in 1 case, closure augmented with a split-thickness skin graft in 2 cases). Nonunion justified revision ORIF and bone grafting in 1 case and 2 fractures required revision ORIF for early fixation failure. Three patients underwent implant removal for symptoms of discomfort. No surgeries were recorded for malunion, amputation, knee fusion, or mobilization under anesthesia. The overall reoperation rate was $12.7 \%$ if implant removal for discomfort was excluded.

Complications were associated with open fractures $(P=$ $0.002)$, although their count was low $(n=5)$. There was no statistical association between complications and sex $(P=$ $0.114)$, age $(P=0.693)$, ISS $(P=0.597)$, number of comorbidities $(P=0.367)$, and time from injury to the operating room $(P=0.221)$.

\section{Radiographic Analysis}

Fractures were classified as 41-C1 in $27(26.5 \%), 41-\mathrm{C} 2$ in $30(29.4 \%)$, and $41-\mathrm{C} 3$ in $45(44.1 \%)$. Calibrated postoperative images were available for all our patients. When analyzing the 4 radiographic criteria used to judge the quality of fracture reduction, the articular reduction was satisfactory (less than 2-mm step or gap) in $63.7 \%$, and a small residual gap was observed in $36.3 \%$. The coronal alignment measured by MPTA was satisfactory ( $87 \pm 5$ degrees) in $97.1 \%$ of the fractures. The sagittal alignment measured by the PPTA was satisfactory ( $9 \pm 5$ degrees) in $96.1 \%$ of the fractures, and a condylar width with $0-5 \mathrm{~mm}$ difference from the femoral condylar width was observed in $96.1 \%$. All 4 criteria were considered satisfactory in 61 fractures $(59.8 \%)$, and 3 or 4 criteria were satisfactory in $95.1 \%$ (Table 2 ).

When we compared our study population with the patients operated after the 72-hour timeframe, we found no statistical association between the timing of surgery and the success rate in obtaining satisfactory radiographic criteria 
TABLE 1. Patients Reoperated Within 12 Months for Complications

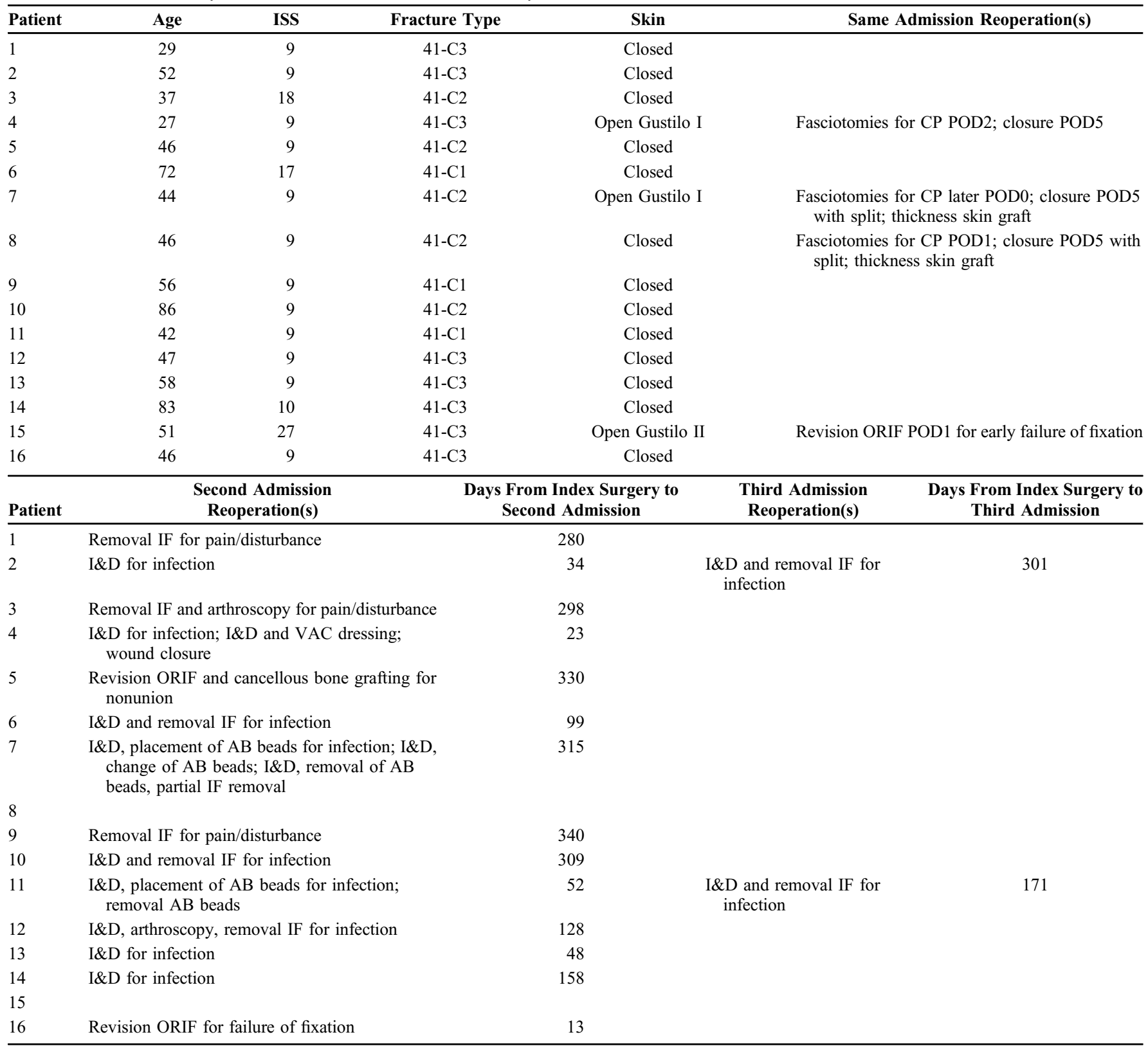

$\mathrm{AB}$, antibiotics; $\mathrm{CP}$, compartment syndrome; I\&D, irrigation and debridement; IF, internal fixation; POD: postoperative day; VAC: vacuum assisted closure.

(articular gap: $P=1.0$, MPTA: $P=1.0$, PPTA: $P=0.106$, condylar width: $P=1.0$ ).

Radiographic follow-up at 12 months was available for all patients, but proper calibration allowing digital measurements was only available in 67 of the 102 fractures $(65.7 \%)$, mostly because patients presented at follow-up from out-oftown with printed radiographs or digitalized images obtained at another institution. Of those, $92.5 \%(\mathrm{n}=62)$ maintained the initial reduction, with the same number of satisfactory radiographic criteria as recorded postoperatively. Reduction was lost in 5 fractures $(7.5 \%)$ and was characterized as medial subsidence in 2 patients, nonunion with deformity in 1 patient, an early hardware removal for infection with subsequent loss of reduction in 1 patient, and 1 conversion to a constrained knee arthroplasty in the context of an associated ipsilateral complex distal femur fracture which failed to heal.

There was no statistically significant difference for age $(P=0.662)$, sex $(P=0.834)$, number of comorbidities $(P=$ $0.592)$, ISS $(P=0.286)$, and fracture classification $(P=$ 0.322 ) between the patients for whom calibrated images were available and the remainder of the cohort. 
TABLE 2. Radiological Reduction in Bicondylar Tibial Plateau Fractures and in the Subset of OTA/AO 41.C3 Type Fractures

\begin{tabular}{|c|c|c|c|c|c|c|}
\hline & \multicolumn{6}{|c|}{ No. Radiographic Criteria Met After ORIF of All OTA/AO 41-C Type Fractures } \\
\hline & 1 & 2 & 3 & 4 & Total & $3+4$ \\
\hline No. cases & 2 & 3 & 36 & 61 & 102 & 97 \\
\hline \multirow[t]{3}{*}{ Percentage of cases } & 2.0 & 2.9 & 35.3 & 59.8 & 100 & 95.1 \\
\hline & \multicolumn{6}{|c|}{ No. Radiographic Criteria Met After ORIF of All OTA/AO 41-C3 Type Fractures } \\
\hline & 1 & 2 & 3 & 4 & Total & $3+4$ \\
\hline No. cases & 1 & 2 & 24 & 18 & 45 & 42 \\
\hline Percentage of cases & 2.2 & 4.5 & 53.3 & 40.0 & 100 & 93.3 \\
\hline
\end{tabular}

\begin{tabular}{|c|c|c|c|c|}
\hline & \multicolumn{4}{|c|}{ Details of Radiographic Criteria Met for OTA/AO 41-C } \\
\hline & $\begin{array}{c}\text { Articular Step or Gap } 2 \mathrm{~mm} \text { or } \\
\text { Less }\end{array}$ & MPTA $87 \pm 5$ degrees & PPTA $9 \pm 5$ degrees & Condylar Width 0-5 mm \\
\hline No. cases & 65 & 99 & 98 & 98 \\
\hline Percentage of cases & 63.7 & 97.1 & 96.1 & 96.1 \\
\hline
\end{tabular}

\begin{tabular}{|c|c|c|c|c|}
\hline & & of Radiographic Criteri & for OTA/AO 41-C3 & \\
\hline & $\begin{array}{c}\text { Articular Step or Gap } 2 \mathrm{~mm} \text { or } \\
\text { Less }\end{array}$ & MPTA $87 \pm 5$ degrees & PPTA $9 \pm 5$ degrees & Condylar Width 0-5 mm \\
\hline No. cases & 19 & 45 & 43 & 42 \\
\hline Percentage of cases & 42.2 & 100.0 & 95.6 & 93.3 \\
\hline
\end{tabular}

\section{Surgical Details}

Seventy-two fractures $(\mathbf{7 0 . 6 \% )}$ were approached using a single lateral incision. Dual incisions combining a lateral and a medial or posteromedial approach were used in 24 fractures $(23.5 \%)$. Other incisions included a single medial approach in 4 cases, a posterior approach in 1 case and an anterior midline incision in 1 early case in our series (Table 3 ). There was a significant association between the severity of the fracture and the choice of approaches: more dual incisions were used in OTA 41-C3 patterns when compared with $\mathrm{C} 1$ and $\mathrm{C} 2$ injuries $(35.6 \%$ vs. $14.0 \%$, $P=0.018)$.

\section{Functional Outcomes}

Functional scores generally improved after 6 months (see Table 4 for details and statistical analysis). At 12 months, the Physical Component of the SF-36 was 42.6, and the DI of the SMFA was 23.4.

TABLE 3. Types of Incisions Used for ORIF

\begin{tabular}{|c|c|c|c|c|}
\hline \multirow{2}{*}{$\begin{array}{l}\text { Type of } \\
\text { Incision }\end{array}$} & \multicolumn{2}{|c|}{$\begin{array}{l}\text { All Bicondylar Fractures } \\
\qquad(N=102)\end{array}$} & \multicolumn{2}{|c|}{$\begin{array}{c}\text { 41-C3 Fractures } \\
(N=45)\end{array}$} \\
\hline & n & $\%$ & $\mathbf{n}$ & $\%$ \\
\hline Lateral & 72 & 70.6 & 25 & 55.6 \\
\hline Dual & 24 & 23.5 & 16 & 35.6 \\
\hline Medial & 4 & 3.9 & 2 & 4.4 \\
\hline Posterior & 1 & 1.0 & 1 & 2.2 \\
\hline Midline & 1 & 1.0 & 1 & 2.2 \\
\hline
\end{tabular}

Minimal skin incisions for placement of a distractor or percutaneous screws are not reported.

\section{DISCUSSION}

High-energy periarticular fractures of the lower extremity present many challenges to the patient and the treating physician. Even in the hands of the most experienced orthopaedic trauma surgeons, these injuries can present technical difficulties at the time of surgery and are often associated with prolonged recovery and high risks of

TABLE 4. Functional Scores for the Subset of Patients Enrolled in the Prospective Study $(n=31)$ : Evolution of the SF-36 and the SMFA

\begin{tabular}{lccc}
\hline & Baseline & 6 Months & 12 Months \\
\hline SF-36 Physical Component & 55.4 & 36.4 & $42.6^{*}$ \\
SF-36 Mental Component & 51.7 & 48.8 & 45.6 \\
SMFA DI & 45.6 & 60.2 & 56.8 \\
SFMA BI & 45.7 & 58.4 & 56.0 \\
SMFA DI & 5.6 & 29.8 & $23.4 \uparrow$ \\
SFMA BI & 5.9 & 31.2 & 25.1 \\
\hline
\end{tabular}

BI, Bother Index.

Standardized Components using norm-based scoring (mean $=50$ ).

Average Individual Standardized Score (higher scores indicate poorer function).

For tibial plateau fractures in particular, values ranging from 18.2 to 22 have been reported in other studies. ${ }^{30-33}$

Repeated analysis of variance (ANOVA) measures determined that mean SF-36 Physical Component scores differed significantly between time points $(P<0.001)$. The differences remained significant after post hoc tests using the Bonferroni correction. For the SF-36 Mental Component, the ANOVA detected significant differences between time points $(P=0.038)$ which did not remain significant after the Bonferroni correction. Comparison of the results of the SMFA DI revealed that after a significant increase at 6 months from baseline $(P<0.001)$, a decrease at 12 months was seen $(P<0.001$; ANOVA: $P<0.001)$. For the SMFA BI, again, after an initial increase at 6 months $(P<0.001)$, a decrease was seen at 12 months $(P<0.001$; ANOVA: $P<0.001)$.

*For comparison, Ahearn et al and Stannard et al ${ }^{34,35}$ both reported a value of 40 in their respective series.

$\dagger$ A clustering around 20 is noted at the 1 year or greater time point and is associated with traumatic conditions in general. ${ }^{36}$ 
complication. The discussion relating to the appropriate treatment for each given fracture is therefore important.

We present a large series of 102 high-energy tibial plateau fractures, suggesting that early definitive ORIF is not necessarily associated with early or late complications when compared with a staged protocol. In our study, $15.7 \%$ of the fractures needed repeat surgery within 12 months for complications (12.7\% if implant removal was excluded). This rate compares favorably with other published results. In the series of 8426 patients operated in Ontario and presented by Henry et al, ${ }^{37} 15.3 \%$ underwent a repeat surgery within 1 year after ORIF for any tibial plateau fracture, regardless of its classification. Morris et al, ${ }^{38}$ in their review of 302 patients with bicondylar fractures, $59.1 \%$ of which were treated with a staged protocol, reported a reoperation rate of $28 \%$ after a follow-up period of 14 months $(21.7 \%$ when excluding implant removal).

In our study, $8.8 \%$ of the fractures developed a wound infection requiring surgical irrigation and debridement. This is comparable to the rates reported in series using staged protocols, which range from $5 \%^{12}$ to $14.2 \%^{38}$ for bicondylar fractures in general, and $8.4 \%{ }^{19}-23.6 \%{ }^{39}$ for $41-\mathrm{C} 3$ type fractures. A randomized trial or matched comparisons would help identifying specific complications associated with early definitive and delayed treatment respectively, in particular with regard to soft tissues and infection, skin necrosis, or compartment syndrome.

Although those results cannot be generalized to the entire study, functional outcome scores obtained after early definitive ORIF in the subset of patients who participated in our prospective data collection were comparable to those presented in other recently published series, in particular for the Physical Component of the SF-36 and the DI of the SMFA at 12 months. ${ }^{30-36}$

Early surgery, performed before hematoma organization, callus formation, and bone resorption, is likely to be associated with easier fracture reduction. It could be argued that the fragility of the soft tissue in early stages might lead the surgeon to choose conservative approaches to the fracture, with a potential impact on the quality of its reduction. In our series, however, early definitive ORIF did not preclude a good quality of fracture reduction based on radiographic criteria (Table 2), and our results parallel those published in other series, in particular when analyzing the subset of patients operated for 41-C3 type fractures. ${ }^{19}$ Although we believe that early surgery allows many bicondylar tibial plateau fractures to be addressed with a single lateral incision augmented withminimal or percutaneous medial approaches, we recognize that fracture classification may not account for differences in fracture patterns, and a specific study aimed to analyze the relationship between the ease and quality of reduction after quantification of initial displacement of the bone fragments, the timing of surgery, and the number of surgical incisions is warranted to confirm our perception.

In our study population, early definitive surgery was associated with an acceptable rate of complications. As only a limited number of fractures were operated after the 72-hour timeframe, our series lacked the power to detect a relationship between delayed surgery and complications. Further study is therefore warranted to specifically explore the relationship between surgical timing and adverse events, as it was done for pilon fractures. ${ }^{40-42}$

Intuitively, treating an injury with 1 , rather than 2 procedures will likely be faster, as it relates to hospital stay and recovery time, and less costly, as long as there is no associated increase in complications. Early ORIF also avoids patient discomfort and pin site infections associated with the use of an external fixator. Formal comparative cost analysis will have to be conducted to corroborate this impression.

We recognize the role for delayed fixation and spanning external fixation in selected cases, but believe that in a center where appropriate resources and surgical expertise are available in a timely fashion, the use of spanning fixators should be limited to those cases where the presence of circumferential blisters, extensive contusion or necrosis, or the general condition of the patient, make early ORIF unreasonable. In our center, this was the case for some $9 \%$ of all tibial plateau fractures during the studied period. This number is not so shocking when one considers that only $50 \%$ of the highenergy C3-type subset of fractures were treated with a staged protocol in the series presented by Barei et al ${ }^{19}$ To our knowledge, no published series follow a well-described protocol which guides decision between early surgery and staged treatment. A future study aimed to identify strictly defined criteria to direct treatment protocols, immediate or delayed, will therefore be very useful.

\section{Limitations of Our Study}

The design of this retrospective review with prospective data collection presents some limitations. Because 14 patients were treated with a knee-spanning device before definitive fixation, our series do not represent consecutive cases of early definitive ORIF. No strictly defined criteria were used to direct choice between primary ORIF and staged protocol, which was made by the treating surgeon.

Properly calibrated radiographic images were only available for $65.7 \%$ of our study population at 12 months. Although there were no statistical differences in the analyzed populations with regard to age, sex, number of comorbidities, ISS, and fracture classification, no definitive conclusions can be drawn as to the stability of fracture reduction in our series.

The functional outcome scores were obtained from a relatively small study population. Although the SF-36 and SMFA scores paralleled other studies, our series lacked the power to underline any statistical associations with factors pertaining to the patients, the type of injury, or the treatment modalities. Comparing the functional results of this study with those reported in the recent literature was furthermore complicated by the use of differing classification schemes, outcome measures, and treatment techniques.

We used the rate of reoperation within 12 months to estimate postoperative complications. This allowed for comparison with other series presented in the literature, but failed to document long-term consequences of primary ORIF such as the development of osteoarthritis and possible conversion to a knee arthroplasty. We did not collect data on complications that did not require reoperation. 


\section{CONCLUSIONS}

Between January 2004 and September 2014, nonstaged operative treatment of bicondylar plateau fractures was performed in $91.3 \%$ of the fractures in our institution. Early definitive ORIF, defined as surgery completed within 72 hours from injury, was performed in $82.3 \%$ of those fractures. In our study population, early definitive ORIF was associated with an acceptable rate of complications. The reoperation rate within 12 months was $15.7 \%$, of which $8.8 \%$ was for infection. The reoperation rate was $12.7 \%$ if implant removal was excluded. Radiographic outcome and functional scores paralleled those published in other series describing staged protocols. Our study suggests that in a model where surgery is performed without delay by experience orthopaedic surgeons, a large proportion of bicondylar tibial plateau fractures can be safely treated with early definitive ORIF, with satisfactory postoperative radiographic reductions.

\section{ACKNOWLEDGMENTS}

The authors acknowledge the invaluable contribution of Ms. Raman Johal, Research Coordinator and Ms. Irene Leung, BScPT, Research Assistant, at the of Division of Orthopaedic Trauma, University of British Columbia.

\section{REFERENCES}

1. Canadian Orthopaedic Trauma Society. Open reduction and internal fixation compared with circular fixator application for bicondylar tibial plateau fractures. Results of a multicenter, prospective, randomized clinical trial. J Bone Joint Surg Am. 2006;88-A:2613-2623.

2. Rademakers MV, Kerkhoffs GM, Sierevelt IN, et al. Operative treatment of 109 tibial plateau fractures: five- to 27-year follow-up results. J Orthop Trauma. 2007;21:5-10.

3. Choo KJ, Morshed S. Postoperative complications after repair of tibial plateau fractures. J Knee Surg. 2014;27:11-20.

4. Rüedi TP, Allgower M. The operative treatment of intra-articular fractures of the lower end of the tibia. Clin Orthop Relat Res. 1979;138:105-110.

5. Mills WJ, Nork SE. Open reduction and internal fixation of high-energy tibial plateau fractures. Orthop Clin North Am. 2002;33:177-198.

6. Moore TM, Patzakis MJ, Harvey JP. Tibial plateau fractures: definition, demographics, treatment rationale, and long-term results of closed traction management or operative reduction. J Orthop Trauma. 1987;1:97-119.

7. Watson JT. High-energy fractures of the tibial plateau. Orthop Clin North Am. 1994;25:723-752.

8. Young MJ, Barrack RL. Complications of internal fixation of tibial plateau fractures. Orthop Rev. 1994;23:149-154.

9. Mallik AR, Covall DJ, Whitelaw GP. Internal versus external fixation of bicondylar tibial plateau fractures. Orthop Rev. 1992;21:1433-1436.

10. Sirkin M, Sanders R, DiPasquale T, et al. A staged protocol for soft tissue management in the treatment of complex pilon fractures. $J$ Orthop Trauma. 2004;18(suppl 8):S32-S38.

11. Tejwani N, Achan P, Staged management of high-energy proximal tibia fractures. Bull Hosp Joint Dis. 2004;62:62-66.

12. Egol KA, Tejwani NC, Capla EL, et al. Staged management of high-energy proximal tibia fractures (OTA types 41 ) the results of a prospective, standardized protocol. J Orthop Trauma. 2005;19:448-455.

13. Benirschke SK, Agnew SG, Mayo KA, et al. Immediate internal fixation of open, complex tibial plateau fractures: treatment by a standard protocol. J Orthop Trauma. 1992;6:78-86.

14. Eggli S, Hartel MJ, Kohl S, et al. Unstable bicondylar tibial plateau fractures: a clinical investigation. J Orthop Trauma. 2008;22:673-679.

15. von Elm E, Altman DG, Egger $M$, et al; STROBE Initiative. The Strengthening the Reporting of Observational Studies in Epidemiology (STROBE) statement: guidelines for reporting observational studies. Lancet. 2007;370:1453-1457.
16. Gustilo RB, Anderson JT. Prevention of infection in the treatment of one thousand and twenty-five open fractures of long bones: a retrospective and prospective analysis. J Bone Joint Surg Am. 1976;58:453-458.

17. Gustilo RB, Mendoza RM, Williams DN. Problems in the management of Type III (severe) open fractures: a new classification of Type III open fracture. J Trauma. 1984;8:742-746.

18. Marsch J, Slongo T, Agel J, et al. Fracture and dislocation classification compendium - 2007: orthopaedic trauma association classification, database and outcomes committee. J Orthop Trauma. 2007;21(suppl 10):S1-S163.

19. Barei DP, Nork SE, Mills WJ, et al. Complications associated with internal fixation of high-energy bicondylar tibial plateau fractures utilising a two-incision technique. J Orthop Trauma. 2004;18:649-657.

20. Blokker CP, Rorabeck CH, Bourne RB. Tibial plateau fractures. An analysis of the results of treatment in 60 patients. Clin Orthop. 1984;73:193-199.

21. Brown TD, Anderson DD, Nepola JV, et al. Contact stress aberrations following imprecise reduction of simple tibial plateau fractures. J Orthop Res. 1988;6:851-862.

22. Honkonen SE. Indications for surgical treatment of tibial condyle fractures. Clin Orthop. 1994;302:199-205.

23. Cooke TD, Li J, Scudamore RA. Radiographic assessment of bony contributions to knee deformity. Orthop Clin North Am. 1994;25:387-393.

24. Paley D. Principles of Deformity Correction. Berlin, German: SpringerVerlag; 2002.

25. Paley D, Herzenberg JE, Tetsworth K, et al. Deformity planning for frontal and sagittal plane corrective osteotomies. Orthop Clin North Am. 1994;25:425-465.

26. Ware JE Jr, Sherbourne CD. The MOS 36-item short-form health survey (SF-36). I. Conceptual framework and item selection. Med Care. 1992; 30:473-483.

27. American Academy of Orthopedic Surgeons (AAOS). Available at: http:// www.aaos.org/research/outcomes/outcomes.asp. Accessed March, 2015.

28. Ponzer S, Skoog A, Bergstrom G. The short musculoskeletal function assessment questionnaire (SMFA): cross-cultural adaptation, validity, reliability and responsiveness of the Swedish SMFA (SMFA-Swe). Acta Orthopaedica Scand. 2003;74:756-763.

29. Swiontkowski MF, Engelberg R, Martin DP, et al. Short musculoskeletal function assessment questionnaire: validity, reliability, and responsiveness. J Bone Joint Surg. 1999;81:1245-1260.

30. Bhattacharyya T, McCarty LP, Harris MB, et al. The posterior shearing tibial plateau fracture: treatment and results via a posterior approach. J Orthop Trauma. 2005;19:305-310.

31. Su E, Westrich G, Rana AJ, et al. Operative treatment of tibial plateau fractures in patients older than 55 years. Clin Orthop. 2004;421:240-248.

32. Abghari M, Marcano A, Davidovitch R, et al. Are locked plates needed for split depression tibial plateau fractures? J Knee Surg. 2016;29:482-486.

33. Haider S, Pean CA, Davidovitch R, et al. Functional outcomes of isolated medial tibial plateau fractures. J Knee Surg. 2015;29:414-422.

34. Ahearn N, Oppy A, Halliday R et al. The outcome following fixation of bicondylar tibial plateau fractures. Bone Joint J. 2014;96-B:956-962.

35. Stannard J, Wilson T, Volgas D, et al. The less invasive stabilization system in the treatment of complex fractures of the tibial plateau: shortterm results. J Orthop Trauma. 2004;18:552-558.

36. Barei D, Agel J, Swiontkowski M. Current utilization, interpretation, and recommendations: the musculoskeletal function assessments (MFA/ SMFA). J Orthop Trauma. 2007;21:738-742.

37. Henry P, Wasserstein D, Paterson M, et al. Risk factors for reoperation and mortality after the operative treatment of tibial plateau fractures in Ontario, 1996-2009. J Orthop Trauma. 2015;29:182-188.

38. Morris BJ, Unger RZ, Archer KR, et al. Risk factors of infection after ORIF of bicondylar tibial plateau fractures. J Orthop Trauma. 2013;27: e196-e200.

39. Ruffolo M, Gettys F, Harvey E, et al. Complications of high-energy bicondylar tibial plateau fractures treated with dual plating through 2 incisions. J Orthop Trauma. 2015;29:85-90.

40. White T, Guy P, Cooke C et al. The results of early primary open reduction and internal fixation for treatment of OTA 43.C-type tibial pilon fractures: a cohort study. J Orthop Trauma. 2010;24:757-763.

41. Wyrsch B, McFerran MA, McAndrew M, et al. Operative treatment of fractures of the tibial plafond. A randomized, prospective study. J Bone Joint Surg Am. 1996;78:1646-1657.

42. Pugh KJ, Wolinsky PR, McAndrew MP, et al. Tibial pilon fractures: a comparison of treatment methods. J Trauma. 1999;47:937-941. 